\title{
On Mildly Nonlinear Partial Difference Equations of Elliptic Type
}

\author{
Lipman Bers ${ }^{2}$
}

\begin{abstract}
The use of the finite differences method is in solving the boundary value problem of the first kind for the nonlinear elliptic equation $\Delta \phi=F\left(x, y, \phi, \phi_{x}, \phi_{y}\right)$ is justified by first showing that the problem of the corresponding difference equation has a unique solution, and then that the solution of the difference equation tends to that of the differential equation when the net unit tends to zero. Also a numerical method of the Liebmann type for the computation of the solution of the difference equation is developed, and these results are extended to more general nonlinear elliptic equations.
\end{abstract}

\section{Introduction}

The method of finite difference is frequently used for the numerical treatment of nonlinear partial differential equations of elliptic type. A theoretical justification of this method, however, seems to exist in the literature only for the case of linear equations. In this note such a justification is given for the simplest nonlinear elliptic equation

$$
\Delta \phi=F\left(x, y, \phi, \phi_{x}, \phi_{y}\right) .
$$

We assume that the partial derivative $F_{\phi}$, is nonnegative and the derivatives $F_{\phi_{x}}, F_{\phi_{y}}$ uniformly bounded, and approximate the differential equation by the difference equation

$\frac{\phi(x+h, y)+\phi(x, y+h)+\phi(x-h, y)+\phi(x, y-h)-4 \phi(x, y)}{h^{2}}$

$$
\begin{array}{r}
=F\left\{x, y, \phi(x, y), \frac{\phi(x+h, y)-\phi(x-h, y)}{2 h},\right. \\
\left.\frac{\phi(x, y+h)-\phi(x, y-h)}{2 h}\right\} .
\end{array}
$$

We shall show that the first boundary value problem for this difference equation possesses a unique solution that can be computed by a Liebmann iteration method, and we shall estimate the difference between this solution and the solution of the boundary value problem for the differential equation.

These results extend almost at once to the case when a more sophisticated difference equation is used to approximate (1), and also, due to a recent result by Motzkin and Wasow, to the elliptic equation

$$
a(x, y) \phi_{x x}+2 b(x, y) \phi_{x y}+\mathrm{c}(x, y) \phi_{y y}=F\left(x, y, \phi, \phi_{x}, \phi_{y}\right) .
$$

Extensions to higher dimensions and to other nets are also immediate. On the other hand, our method is tied to the maximum-principle; it does not apply

\footnotetext{
1 The preparation of this paper was sponsored (in part) by the Office of Naval Research.

2 Present address New York University, New York, N. Y.
}

to such important quasi-linear equations as the equation of minimal surfaces or the equations of gas dynamics.

We state explicitly that the reasoning reproduced below involves only a straightforward application of familiar arguments. In section 3 , in particular, we paraphrase well-known facts in a form suitable for our purposes.

\section{Notations}

We denote by $\Omega$ a fixed bounded domain in the $(x, y)$-plane. $\bar{\Omega}$ is the closure and $\Omega^{\prime}$ the boundary of ת. $F(x, y, z, p, q)$ denotes a fixed continuous function defined for $(x, y) \epsilon \bar{\Omega}$ and all $z, p, q$. We assume that the partial derivatives $F_{2}, F_{p}, F_{q}$ exist and satisfy the inequalities

$$
\begin{gathered}
\mathrm{F}_{z} \geq 0, \\
\text { Set } \quad\left|F_{p}\right|,\left|F_{q}\right| \leq A<+\infty . \\
\boldsymbol{L}[\phi]=\Delta \phi-F\left(x, y, \phi, \phi_{x}, \phi_{y}\right),
\end{gathered}
$$

where $\Delta$ is the Laplace operator $\left(\Delta \phi=\phi_{x x}+\phi_{y y}\right)$. Let $v(x, y)$ be a fixed continuous function defined on $\bar{\Omega}$. Boundary value problem $\boldsymbol{P}$ consists in finding a function $\phi(x, y)$ continuous on $\bar{\Omega}$ and twice continuously differentiable in $\Omega$ such that

$$
\boldsymbol{L}[\phi]=0 \text { in } \Omega, \quad \phi=v \text { on } \Omega^{\prime} .
$$

It is known that this problem has at most one solution, and that a solution exists under appropriate smoothness hypotheses.

We approximate $\boldsymbol{L}$ by a difference operator $\boldsymbol{L}_{n}$ defined (for $h>0$ ) by

$$
\boldsymbol{L}_{h}[\phi]=\Delta_{h}[\phi]-F\left\{x, y, \phi, D_{h, x}[\phi], D_{h, y}[\phi]\right\},
$$

where

$$
\begin{aligned}
& \Delta_{h}[\phi(x, y)]= \\
& \frac{\phi(x+h, y)+\phi(x, y+h)+\phi(x-h, y)+\phi(x, y-h)-4 \phi(x, y)}{h^{2}},
\end{aligned}
$$




$$
\begin{aligned}
& D_{h, x}[\phi(x, y)]=\frac{\phi(x+h, y)-\phi(x-h, y)}{2 h}, \\
& D_{h, x}[\phi(x, y)]=\frac{\phi(x, y+h)-\phi(x, y-h)}{2 h} .
\end{aligned}
$$

Let $P_{0}$ be the point with the coordinates $\left(x_{0}, y_{0}\right)$. The points $\left(x_{0}+h, y_{0}\right),\left(x_{0}, y_{0}+h\right),\left(x_{0}-h, y_{0}\right),\left(x_{0}, y_{0}-h\right)$ will be called the $h$-neighbors of $P_{0}$ and will be denoted by $P_{01}, \ldots, P_{04}$. A lattice domain $\Omega_{h}$ is a set of points, $P_{1}, \ldots, P_{N}$, situated in $\Omega$, having coordinates that are integral multiples of $h$, and such that all points $P_{i \nu}, i=1, \ldots, N ; \nu=1, \ldots, 4$ belong to $\bar{\Omega}$. Neighbors of points of $\Omega_{h}$ that are not themselves points of $\Omega_{h}$ form the boundary $\Omega_{h}^{\prime}$ of $\Omega_{h}$. We denote the points of $\Omega_{h}^{\prime}$ by $P_{N+1}, \ldots, P_{M}$, and the union of $\Omega_{h}$ and $\Omega_{h}^{\prime}$ by $\bar{\Omega}_{h}$. If $\Omega_{h}$ is fixed, and $\chi$ is a function defined on $\bar{\Omega}_{h}$, we denote the value of $\chi$ at a point $P_{i}$ (or at a point $\left.P_{i v}\right)$ by $\chi_{i}\left(\right.$ or $\left.\chi_{i v}\right)$. We also set

$$
\boldsymbol{L}_{h}\left[\boldsymbol{\phi}_{i}\right]=\Delta_{h}\left[\boldsymbol{\phi}_{i}\right]-F\left\{x_{i}, y_{i}, \boldsymbol{\phi}_{i}, D_{h, x}\left[\phi_{i}\right], D_{h, y}\left[\phi_{i}\right]\right\},
$$

where

$$
\begin{gathered}
\Delta_{h}\left[\phi_{i}\right]=\frac{1}{h^{2}}\left\{\sum_{\nu=1}^{4} \phi_{i \nu}-4 \phi_{i}\right\} \\
D_{h, x}\left[\phi_{i}\right]=\frac{\phi_{i 1}-\phi_{i 3}}{2 h}, \quad D_{h, y}\left[\phi_{i}\right]=\frac{\phi_{i 2}-\phi_{i 4}}{2 h} .
\end{gathered}
$$

The boundary-value problem $\boldsymbol{P}_{h}$ (for some fixed $\Omega_{h}$ ) consists in determining a function $\phi$ defined on $\bar{\Omega}_{h}$ such that

$$
\begin{aligned}
\boldsymbol{L}_{h}\left[\phi_{i}\right]=0, \quad i= & 1, \ldots, N ; \\
& \phi_{j}=v_{j}, \quad j=N+1, \ldots, M .
\end{aligned}
$$

From now on we assume that $h$ is so small that at least one $\Omega_{h}$ exists, and that

$$
A h<2 \theta, \quad 0<\theta<1,
$$

where $\theta$ is some fixed number.

\section{An Inequality}

In this section we recall some properties of the linear difference operator

$$
l_{h}\left[\phi_{i}\right]=\Delta_{h}\left[\phi_{i}\right]+\alpha_{i} D_{h, x}\left[\phi_{i}\right]+\beta_{i} D_{h, y}\left[\phi_{i}\right]-\gamma_{i} \phi_{i},
$$

with

$$
\begin{gathered}
\left|\alpha_{i}\right|,\left|\beta_{i}\right| \leq A, \\
\gamma_{i} \geq 0 .
\end{gathered}
$$

In particular, we want to establish the existence of a constant

$$
C=C(A, \theta, d),
$$

depending only on $A, \theta$ and the diameter $d$ of $\Omega$ such that the inequality

$$
\max _{\Omega_{h}}|\phi| \leq C \max _{\Omega_{h}}\left|l_{h}[\phi]\right|+\max _{\Omega_{h}^{\prime}}|\phi|
$$

holds for every function $\phi$ defined on $\bar{\Omega}_{h}$.

We observe first that the equation

$$
l_{h}\left[\phi_{i}\right]=0, \quad i=1, \ldots, N,
$$

may be written in the form

$$
\phi_{i}=\frac{\sum_{\nu=1}^{4} \lambda^{i_{\nu}} \phi_{i \nu}}{1+\frac{h^{2}}{4} \gamma_{i}}, \quad i=1, \ldots, N
$$

where

$$
\begin{aligned}
\lambda^{i 1} & =\frac{1}{4}\left(1+\frac{h}{2} \alpha_{i}\right), & \lambda^{i 2} & =\frac{1}{4}\left(1+\frac{h}{2} \beta_{i}\right), \\
\lambda^{i 3} & =\frac{1}{4}\left(1-\frac{h}{2} \alpha_{i}\right), & \lambda^{i 4} & =\frac{1}{4}\left(1-\frac{h}{2} \beta_{i}\right) .
\end{aligned}
$$

Since

$$
\lambda^{i_{\nu}} \geq \frac{1}{4}(1-\theta)>0, \quad \sum_{\nu=1}^{4} \lambda^{i_{\nu}}=1,
$$

(18) implies that

$$
\phi_{i}\left\{\begin{array}{lll}
\leq \max \left(\phi_{i 1}, \ldots, \phi_{i 4}\right) & \text { if } & \phi_{i} \geq 0 \\
\geq \min \left(\phi_{i 1}, \ldots, \phi_{i 4}\right) & \text { if } & \phi_{i} \leq 0
\end{array}\right.
$$

and the equality sign in (19) holds only if $\phi_{i l}=$. . $=\phi_{i 4}, \gamma_{i} \phi_{i}=0$.

Let $m$ and $M$ be the minimum and maximum of a solution of (17). If $M>0$ and $\phi=M$ at a point $P_{i}$ of $\Omega_{h}$, then $\varphi=M$ at all neighbors of $P_{i}$, at all neighbors of neighbors, etc. Hence $\phi=M$ at a point of $\Omega_{h}^{\prime}$. A similar argument holds if $m<0$. Thus

$$
\min \left(0, \min _{\Omega_{h}^{\prime}}^{\prime} \phi\right) \leq \phi_{i} \leq \max \left(0, \max _{\Omega_{h}^{\prime}} \varphi\right), \quad i=1, \ldots, N,
$$

and, in particular,

$$
\max _{\Omega_{h}}|\phi| \leq \max _{\Omega_{h}^{\prime}}|\varphi| .
$$

Consider now the boundary value problem

$$
\begin{aligned}
l_{h}\left[\phi_{i}\right]=k_{i}, \quad i=1, \ldots, & N ; \\
\phi_{j} & =v_{j}, \quad j=N+1, \ldots, M .
\end{aligned}
$$

It is equivalent to the system

$$
\sum_{s=1}^{N} L^{i s} \phi_{s}=k_{i}+\sum_{j=N+1}^{M} \sigma^{i j} v_{j}, \quad i=1, \ldots, N,
$$

where the matrices $\left(L^{i s}\right)$ and $\left(\sigma^{i j}\right)$ depend only on $l_{h}$ and $\Omega_{h}$. In view of (21) the homogeneous system $(k \equiv 0, v \equiv 0)$ has only the trivial solution. Hence the "Green matrix" $\left(G^{i j}\right)=\left(L^{i j}\right)^{-1}$ of $l_{h}$ in $\Omega_{h}$ exists, and the unique solution of (22) is given by

$$
\phi_{i}=\sum_{s=1}^{N} G^{i s} k_{s}+\sum_{j=N+1}^{M} \Gamma^{i j} v_{j}, \quad i=1, \ldots, N,
$$


where

$$
\Gamma^{i j}=\sum_{l=1}^{N} G^{i l} \sigma^{l j}
$$

we call $\left(\Gamma^{i j}\right)$ the "Green boundary matrix" of $l_{h}$. Thus every function defined on $\bar{\Omega}_{h}$ satisfies the identity

$$
\phi_{i}=\sum_{s=1}^{N} G^{i s} l_{h}\left[\phi_{s}\right]+\sum_{j=N+1}^{M} \Gamma^{i j} \phi_{j}, \quad i=1, \ldots, N,
$$

and hence the inequality

$$
\max _{\Omega_{h}}|\phi| \leq c_{1} \max _{\Omega_{h}}\left|l_{h}[\phi]\right|+c_{2} \max _{\Omega_{h}^{\prime}}|\phi|
$$

with

$$
c_{1}=\max \sum_{s=1}^{N}\left|G^{i s}\right|, \quad c_{2}=\max \sum_{j=N+1}^{M}\left|\Gamma^{i j}\right| .
$$

Let $\phi$ be a solution of (22) with $k_{i} \equiv 0, v_{j}=\delta_{j l}$ (Kronecker $\delta$ ). Then $\phi_{i}=\Gamma^{i l}$. By property (20) of solutions of (17), $0 \leq \phi_{i} \leq 1, i=1,2, \ldots, N$. Thus $\Gamma^{i l} \geq 0$ for all $i, l$. Next let $\phi$ be a solution of (22) with $k_{i} \equiv 0, v_{N+1}=\ldots=v_{M}=1$. Then

$$
\phi_{1}=\sum_{j=N+1}^{M} \Gamma^{i j}=\sum_{j=N+1}^{M}\left|\Gamma^{i j}\right|,
$$

and by property (20) of solutions of (17), $0 \leq \phi_{i} \leq 1$. Hence,

$$
c_{2} \leq 1 \text {. }
$$

Now let $\phi$ be the solution of (22) with $k_{i}=\delta_{i l}, v_{j} \equiv 0$, so that $\phi_{i}=G^{i z}$. Let $\dot{\Omega}_{h}$ denote the lattice domain obtained from $\Omega_{h}$ by removing $P_{l}$. Assume that $\phi_{l}>0$. In $\dot{\Omega}_{h}$ we have that $l_{h}[\phi]=0$, so that by $(20)$ $0 \leq \phi_{i} \leq \phi_{l}, i \neq l$. In particular, $0 \leq \phi_{l \nu} \leq \phi_{l}, \nu=1,2,3,4$. But $l_{h}\left[\phi_{l}\right]=1$, so that

$$
\frac{h^{2}}{4}+\left(1+\frac{h^{2}}{4} \gamma_{l}\right) \phi_{l}=\sum_{\nu=1}^{4} \lambda^{l_{\nu}} \phi_{l \nu} \leq \phi_{l}
$$

which is absurd. Hence $\phi_{l} \leq 0$, and again by (20) $\phi_{i} \leq 0$ for $i \neq l$. Hence

$$
G^{i l} \leq 0
$$

Now set

$$
\widetilde{l}_{h}\left[\phi_{i}\right]=\Delta_{h}\left[\phi_{i}\right]+\alpha_{i} D_{h, x}\left[\phi_{i}\right]+\beta_{i} D_{h, y}\left[\phi_{i}\right]
$$

and let $\widetilde{G}^{i l}, \widetilde{\Gamma}^{i l}, \widetilde{c}_{1}, \widetilde{c}_{2}$ be defined as before, with $l_{h}$ replaced by $\widetilde{l}_{h}$. Let $\phi$ have the same meaning as before, and let $\widetilde{\phi}$ be the solution of the boundary value problem

$\widetilde{l_{h}}\left[\widetilde{\phi_{i}}\right]=\delta_{\imath l}, \quad i=1, \ldots, N$;

$$
\widetilde{\phi}_{y}=0, \quad j=N+1, \ldots, M \text {. }
$$

Then

$$
\widetilde{\phi}_{i}=\widetilde{\Gamma}^{i l} \leq 0
$$

and since for $i=1, \ldots, N$

$$
\begin{gathered}
l_{h}\left[\phi_{i}-\widetilde{\phi}_{i}\right]=l_{h}\left[\phi_{i}\right]-\left\{\widetilde{l}_{h}\left[\widetilde{\phi}_{i}\right]-\gamma_{i} \widetilde{\phi}_{i}\right\}=\gamma_{i} \widetilde{\phi}_{i} \leq 0, \\
\phi_{i}-\widetilde{\phi}_{i}=\sum_{s=1}^{N} G^{i s} \gamma_{s} \widetilde{\phi}_{s} \geq 0 .
\end{gathered}
$$

Thus $\widetilde{G}^{i l} \leq G^{i l}$, or $\left|G^{i l}\right| \leq\left|\widetilde{G}^{i l}\right|$ so that

$$
c_{1} \leq \widetilde{c}_{1} .
$$

Let $\Phi(x, y)$ be a continuous positive function defined on $\bar{\Omega}$ for which $\widetilde{l}_{h}[\Phi]>0$, and

$$
\frac{\max _{\Omega}^{-} \Phi}{\min _{\Omega_{h}} \widetilde{l}_{h}[\Phi]} \leq C(A, \theta, d) .
$$

We have that

$$
\Phi_{i}=\sum_{s=1}^{N} \widetilde{G}^{i} \widetilde{l}_{h}\left[\Phi_{s}\right]+\sum_{j=N+1}^{M} \widetilde{\Gamma}^{i j} \Phi_{j}, \quad i=1, \ldots, N,
$$

and as the term on the left-hand side is positive,

$$
\sum_{s=1}^{N}\left|\widetilde{G}^{i s}\right| \widetilde{l}_{h}\left[\Phi_{s}\right] \leq \sum_{j=N+1}^{M} \widetilde{\Gamma}^{i j} \Phi_{j} .
$$

Noting that $\widetilde{c}_{2} \leq 1$, we obtain the inequality

$$
\left\{\min _{\Omega_{h}} l_{h}[\Phi]\right\} \sum_{s=1}^{N}\left|\widetilde{G}^{i s}\right| \leq \max _{\bar{\Omega}} \Phi,
$$

which implies that

$$
\widetilde{c}_{1} \leq C .
$$

From (23), (25), (28) and (30) the assertion (16) follows.

We conclude the proof by exhibiting a function $\Phi(x, y)$ having the desired property (without aiming at the best possible value of $C$ ). Without loss of generality we assume that $\Omega$ is located within the strip $0 \leq x \leq d$.

If $A \bar{d}<\overline{2}$, we set $\Phi(x, y)=[x-(d / 2)]^{2}$. Then

$$
\Delta_{h}[\Phi]=2, \quad D_{h, x}[\Phi]=2 x-d, \quad D_{h, y}[\Phi]=0,
$$

so that in $\bar{\Omega}: \widetilde{l}_{h}[\Phi] \geq 2-A d$, and of course $\Phi \leq d^{2} / 4$. Hence in this case (29) holds with $C=d^{2} / 4[2-A d]$. If $A d>2$, set

$$
\mu=\frac{A}{\theta} \tanh ^{-1}\left(\frac{3 \theta-\theta^{2}}{2}\right), \quad \Phi(x, y)=e^{\mu x} .
$$

Then $\max _{\bar{\Omega}}^{-} \Phi \leq e^{\mu d}$ and

$$
\Delta_{h}[\Phi]=4 e^{\mu x}\left[\frac{\sinh (\mu h / 2)}{h}\right]^{2},
$$

$D_{h, x}[\Phi]=2 e^{\mu x}\left[\frac{\sinh (\mu h / 2)}{h}\right] \cosh (\mu h / 2), \quad D_{h, y}[\Phi]=0$, 
so that

$$
\widetilde{l}_{h} \geq 2 e^{\mu x} \mu \frac{\sinh (\mu h)}{\mu h}\left[\frac{\tanh (\mu h / 2)}{h}-\frac{A}{2}\right] .
$$

Because $(\tanh \xi) / \xi$ is a decreasing function of $\xi$, the expression in the brackets is, for $0 \leq h \leq 2 \theta / A$, not less than

$$
\frac{\tanh (\mu \theta / A)}{2 \theta / A}-\frac{A}{2} \leq \frac{A}{4}(1-\theta),
$$

so that

$$
\tilde{l}_{h}[\Phi] \geq \frac{A^{2}(1-\theta)}{2 \theta} \tanh ^{-1}\left(\frac{3 \theta-\theta^{2}}{2}\right) .
$$

Thus (29) holds again with

$$
C=\frac{2 \theta \exp \left\{\frac{A d}{\theta} \tanh ^{-1}\left(\frac{3 \theta-\theta^{2}}{2}\right)\right\}}{A^{2}(1-\theta) \tanh ^{-1}\left(\frac{3 \theta-\theta^{2}}{2}\right)} .
$$

\section{Existence and Uniqueness of the Solution of the Difference Equation}

The inequality

$$
\begin{aligned}
\max _{\Omega_{h}}\left|\phi^{\mathrm{I}}-\phi^{\mathrm{II}}\right| \leq C \max _{\Omega_{h}} \mid \boldsymbol{L}_{h}\left[\phi^{\mathrm{I}}\right] & -\boldsymbol{L}_{h}\left[\phi^{\mathrm{II}}\right] \mid \\
& +\max _{\Omega_{h}^{\prime}}\left|\phi^{\mathrm{I}}-\phi^{\mathrm{II}}\right|
\end{aligned}
$$

holds for any two functions defined on $\bar{\Omega}_{h}$.

In fact, by the mean-value theorem, the function $\phi=\phi^{\mathrm{I}}-\phi^{\mathrm{II}}$ satisfies the difference equation

$$
l_{h}[\phi]=\boldsymbol{L}_{h}\left[\phi^{\mathrm{I}}\right]-\boldsymbol{L}_{h}\left[\phi^{\mathrm{II}}\right],
$$

where $l_{h}$ is defined by (12) with

$$
\begin{gathered}
\alpha_{i}=-F_{p}\left(x_{i}, y_{i}, z_{i}, p_{i}, q_{i}\right), \quad \beta_{i}=-F_{q}\left(x_{i}, y_{i}, z_{i}, p_{i}, q_{i}\right), \\
\gamma_{i}=F_{z}\left(x_{i}, y_{i}, z_{i}, p_{i}, q_{i}\right), \quad z_{i}=\tau_{i} \varphi_{i}^{\mathrm{I}}+\left(1-\tau_{i}\right) \phi_{i}^{\mathrm{II}}, \\
p_{i}=\frac{1}{2 h}\left\{\tau_{i}\left(\phi_{i 1}^{\mathrm{I}}-\phi_{i 3}^{\mathrm{I}}\right)+\left(1-\tau_{i}\right)\left(\phi_{i 1}^{\mathrm{II}}-\phi_{i 3}^{\mathrm{II}}\right)\right\}, \\
q_{i}=\frac{1}{2 h}\left\{\tau_{i}\left(\phi_{i 2}^{\mathrm{I}}-\phi_{i 4}^{\mathrm{I}}\right)+\left(1-\tau_{i}\right)\left(\phi_{i 2}^{\mathrm{II}}-\phi_{i 4}^{\mathrm{II}}\right)\right\},
\end{gathered}
$$

the $\tau_{i}$ being numbers such that $0 \leq \tau_{i} \leq 1$. Since conditions (13), (14) are satisfied, inequality (16) is applicable and yields (31).

It follows from (31) that problem $\boldsymbol{P}_{h}$ has at most one solution.

Set

$$
C_{2}=\max _{\bar{\Omega}}^{-}|F(x, y, 0,0,0)| .
$$

Then $\left|\boldsymbol{L}_{h}(0)\right| \leq C_{2}$, so that applying (31) to an arbitrary function $\phi=\phi^{\mathrm{I}}$ and to the function $\phi^{\mathrm{II}} \equiv 0$, we obtain

$$
\max _{\Omega_{h}}|\phi| \leq C C_{2}+C \max _{\Omega_{h}}\left|\boldsymbol{L}_{h}[\phi]\right|+\max _{\Omega_{h}^{\prime}}|\phi|
$$

for every function defined on $\bar{\Omega}_{h}$.

In particular, a solution $\phi$ of $\boldsymbol{P}_{h}$ satisfies the inequality

$$
\max _{\Omega_{h}}|\phi| \leq K=C C_{2}+\max _{\bar{\Omega}}^{-}|v| .
$$

Now let $\left(G^{i s}\right)$ and $\left(\Gamma^{i j}\right)$ be the Green matrix and the Green boundary matrix of the operator $\Delta_{h}$ for the domain $\Omega_{h}$, and define the continuous transformation $\vec{\phi}^{*}=T_{t}(\vec{\phi})$ in the $N$-dimensional $\left(\phi_{1}, \ldots, \varphi_{N}\right)$ space by the equations

$$
\begin{aligned}
\phi_{i}^{*}=\phi_{i}-t \sum_{s=1}^{N} G^{i s} F & \left\{x_{i}, y_{i}, \phi_{i}, D_{h, x}\left[\phi_{i}\right], D_{h, y}\left[\phi_{i}\right]\right\} \\
& -\sum_{j=N+1}^{M} \Gamma^{i j} v_{j}, \quad i=1, \ldots, N,
\end{aligned}
$$

where $t$ is a real parameter, $0 \leq t \leq 1$. A point $\vec{\phi}=\left(\phi_{1}, \ldots, \varphi_{N}\right)$ taken by this transformation into the origin is a solution of the boundary value problem

$$
\begin{aligned}
& \Delta_{h}\left[\phi_{i}\right]-t F\left\{x_{i}, y_{i}, \phi_{i}, D_{h, x}\left[\phi_{i}\right], D_{h, y}\left[\phi_{i}\right]\right\}=0, \\
& i=1, \ldots ., N, \quad \phi_{j}=v_{j}, \quad j=N+1, \ldots, M .
\end{aligned}
$$

Let $\bar{S}$ denote the domain $\phi_{1}^{2}+\ldots+\phi_{N}^{2} \leq N K^{2}+1$. By virtue of (34) no point of the boundary $S^{\prime}$ of $\bar{S}$ is taken into the origin by $T_{t}$. Hence the degree of the mapping $T_{t}$ of $\bar{S}$ at the origin (that is the $N$-dimensional solid angle under which the image $T_{t}\left(S^{\prime}\right)$ is seen from the origin, divided by the measure of the $N$-dimensional unit sphere) is well defined. Since $T_{t}$ depends continuously on $t$, this degree is independent of $t$. For $t=0$ the mapping $T_{0}$ is a translation which takes the point $\overrightarrow{\phi^{0}}$, with $\phi_{i}^{0}=\sum_{j=N+1}^{M} \Gamma^{i j} v_{j}$ of $\bar{S}$ into the origin. Hence the degree is 1 . Thus for $t=1$ at least one point $\vec{\phi}$ of $\bar{S}$ satisfies $T_{1}(\vec{\phi})=0$, and problem $\boldsymbol{P}_{h}$ has a solution.

A somewhat longer but constructive existence proof will be given later.

\section{Convergence of the Solution of the Differ- ence Equation to that of the Differential Equation}

We assume now that problem $\boldsymbol{P}$ has a solution $\phi(x, y)$.

Let $\omega(\eta)$ be the modulus of continuity of $\phi(x, y)$ in $\bar{\Omega}$, that is,

$$
\begin{aligned}
\omega(\eta)= & \text { l.u.b. }\left|\phi(x, y)-\phi\left(x^{\prime}, y^{\prime}\right)\right| \text { for } \\
& (x, y),\left(x^{\prime}, y^{\prime}\right) \epsilon \bar{\Omega}, \quad\left(x-x^{\prime}\right)^{2}+\left(y-y^{\prime}\right)^{2} \leq \eta^{2} .
\end{aligned}
$$

By $\omega_{\delta}^{(2)}(\eta), m_{\delta}^{(2)}$ we denote the maximum of the moduli of continuity and of the absolute values, respectively, of the second derivatives of $\phi(x, y)$ in the closed subdomain of $\Omega$ consisting of all points in $\Omega$ having a distance not less than $\delta>0$ from $\Omega^{\prime} . m_{\delta}^{(2)}$ and (for a fixed $\eta>0) \omega_{\delta}^{(2)}(\eta)$ are nonincreasing functions 
of $\delta$. Set

$$
\lim _{\delta \rightarrow 0} m_{\delta}^{(2)}=m^{(2)}, \quad \lim _{\delta \rightarrow 0} \omega_{\delta}^{(2)}(\eta)=\omega^{(2)}(\eta) .
$$

If the second derivatives of $\phi(x, y)$ are continuous in $\bar{\Omega}$, then $m^{(2)}<+\infty$ and $\omega_{\delta}^{(2)}(\eta) \rightarrow 0$ for $\eta \rightarrow 0$. If $\phi(x, y)$ possesses continuous partial derivatives of the third or fourth order, we define $m_{\delta}^{(3)}, m^{(3)}, m_{\delta}^{(4)}, m^{(4)}$ in a similar manner. Finally, we denote by $\omega^{*}(\eta)$ the modulus of continuity of $v(x, y)$ in $\bar{\Omega}$.

Let $\Omega_{h}$ be a lattice domain such that (i) every point of $\Omega_{h}^{\prime}$ has a distance not exceeding $\epsilon$ from some point of $\Omega^{\prime}$, (ii) $\bar{\Omega}_{h}$ is contained in a closed subdomain of $\Omega$ having a distance not less than $\delta>0$ from $\Omega^{\prime}$. We denote by $\phi^{(h, \epsilon, \delta)}$ the solution of problem $\boldsymbol{P}_{h}$ for this domain.

Let $P_{j}$ be a point of $\Omega_{h}^{\prime}$. By virtue of (i) there exists a point $(x, y)$ of $\Omega^{\prime}$ such that

$$
\left|v_{j}-v(x, y)\right| \leq \omega^{*}(\boldsymbol{\epsilon}), \quad\left|\phi_{j}-\phi(x, y)\right| \leq \omega(\boldsymbol{\epsilon}) .
$$

Since $\phi_{j}^{(h, \epsilon, \delta)}=v_{j}, \quad \phi(x, y)=v(x, y)$, we have that

$$
\max _{\Omega \Omega_{h}^{\prime}}\left|\phi^{(h, \epsilon, \delta)}-\phi\right| \leq \omega(\epsilon)+\omega^{*}(\epsilon) .
$$

From Taylor's formula and condition (ii) it follows easily that for every point $P_{i}$ of $\Omega_{h}$,

$$
\left|D_{h, x}\left[\phi_{i}\right]-\left(\phi_{x}\right)_{i}\right|,\left|D_{h, y}\left[\phi_{i}\right]-\left(\phi_{y}\right)_{i}\right| \leq m_{\delta}^{(2)} h,
$$

so that

$\mid F\left\{x_{i}, y_{i}, \phi_{i}, D_{h, x}\left[\phi_{i}\right], D_{h, y}\left[\phi_{i}\right]\right\}$

Also $-\left\{F\left(x, y, \phi, \phi_{x}, \phi_{y}\right)\right\}_{i} \mid \leq 2 A m_{\delta}^{(2)} h$.

$$
\left|\Delta_{h}\left[\phi_{i}\right]-(\Delta \phi)_{i}\right| \leq 2 \omega_{\delta}^{(2)}(h) \text {. }
$$

Since $\boldsymbol{L}[\phi] \equiv 0$,

$$
\max _{\Omega_{h}}\left|\boldsymbol{L}_{h}[\phi]\right| \leq 2 A m_{\delta}^{(2)} h+2 \omega_{\delta}^{(2)}(h) .
$$

Inequality (31) now yields the estimate

$$
\begin{aligned}
\max _{\Omega_{h}}\left|\phi^{(h, \epsilon, \delta)}-\phi\right| \leq 2 C\left[m_{\delta}^{(2)} h\right. & \\
& \left.+\omega_{\delta}^{(2)}(h)\right]+\omega(\epsilon)+\omega^{*}(\epsilon) .
\end{aligned}
$$

'This relation shows that $\phi$ can be approximated by $\phi^{(h, \epsilon, \delta)}$ with any desired degree of accuracy. In fact, let $\eta<0$ be given. We determine an $\epsilon<0$ so that $\omega(\epsilon)+\omega^{*}(\epsilon) \leq \eta / 2$ and choose a closed subdomain $\bar{\Omega}^{*}$ of $\Omega$ such that every boundary point of $\bar{\Omega}^{*}$ has a distance not exceeding $\epsilon$ from some point of $\Omega^{\prime}$. For every $h>0$ let $\Omega_{h}$ consist of all points in the interior $\Omega^{*}$ of $\bar{\Omega}^{*}$ whose coordinates are integral multiples of $h$ and whose $h$-neighbors belong to $\bar{\Omega}^{*}$. Let $\delta$ be the (positive) distance of $\bar{\Omega}^{*}$ to $\Omega^{\prime}$. We choose $h$ so small that $2 C\left[m_{\delta}^{(2)} h+\omega_{\delta}^{(2)}(h)\right] \leq \eta / 2$. Then
If the partial derivatives of $\phi(x, y)$ of the third order exist and are continuous in $\Omega$, we may replace (38), (39) by

$$
\begin{gathered}
\left|D_{h, x}\left[\phi_{i}\right]-\left(\phi_{x}\right)_{i}\right|,\left|D_{h, y}\left[\phi_{i}\right]-\left(\phi_{y}\right)_{i}\right| \leq \frac{1}{6} m_{\delta}^{(3)} h^{2}, \\
\left|\Delta_{h}\left[\phi_{i}\right]-(\Delta \phi)_{i}\right| \leq \frac{2}{3} m_{\delta}^{(3)} h,
\end{gathered}
$$

and instead of (40) we obtain the estimate

$$
\max _{\Omega_{h}}\left|\phi^{(h, \epsilon, \delta)}-\phi\right| \leq \frac{1}{3} C m_{\delta}^{(3)}\left(A h^{2}+2 h\right)+\omega(\epsilon)+\omega^{*}(\epsilon) .
$$

If $\phi(x, y)$ possesses continuous derivatives of order four, $\left(39^{\prime}\right)$ may be replaced by

$$
\left|\Delta_{h}\left[\phi_{i}\right]-(\Delta \phi)_{i}\right| \leq \frac{1}{6} m_{\hat{\delta}}^{(4)} h^{2},
$$

so that instead of $\left(40^{\prime}\right)$ we obtain

$\max _{\Omega_{h}}\left|\phi^{(h, \epsilon, \delta)}-\phi\right| \leq \frac{1}{6} C\left(2 m_{\delta}^{(3)}+m_{\delta}^{(4)}\right) h^{2}+\omega(\epsilon)+\omega^{*}(\epsilon)$.

If the derivatives of $\phi(x, y)$ (of order $2,3,4$, respectively) are continuous in $\bar{\Omega}$, condition (ii) is superfluous. We may drop the superscript and subscript $\delta$ in $(40),\left(40^{\prime}\right),\left(40^{\prime \prime}\right)$ and obtain, for $h \rightarrow 0, \epsilon \rightarrow 0$, uniform convergence of $\phi^{(h, \epsilon)}$ to $\phi$. In fact if all points of $\Omega_{h}^{\prime}$ are on $\Omega^{\prime}$ the terms with $\epsilon$ disappear and we have, say in the case $m^{(4)}<+\infty$, that $\left|\phi^{(h)}-\phi\right|=0\left(h^{2}\right)$.

All preceding estimates involve $(\alpha)$, some a priori estimates for the solution of $\boldsymbol{P},(\beta)$ the exact solution of $\boldsymbol{P}_{h}$. Without some a priori information on the solution of the differential equation, however, its approximation by the solution of the difference equation can never be estimated. On the other hand, the difference between an exact and an approximate solution of the difference equation (and only approximate solutions can be obtained in practice) can be estimated at once by means of (31).

\section{Solution of the Difference Equation by Iterations}

In this section we describe an effective method for solving problem $\boldsymbol{P}_{h}$, and at the same time obtain a constructive existence proof.

Define (for $i=1,2, \ldots, N$ )

$f_{i}\left(z ; \zeta^{1}, \zeta^{2}, \zeta^{3}, \zeta^{4}\right)=z+\frac{h_{2}}{4} F\left(x_{i}, y_{i}, z, \frac{\zeta^{1}-\zeta^{3}}{2 h}, \frac{\zeta^{2}-\zeta^{4}}{2 h}\right)$

Then

$$
\frac{\partial f_{i}}{\partial z}=1+\frac{h_{2}}{4} F_{z}
$$

is nonnegative, so that there exists functions

$$
z=g_{i}\left(Z ; \xi^{1}, \ldots, \xi^{4}\right)
$$


such that

$$
f_{i}\left[g_{i}\left(Z ; \zeta^{1}, \ldots, \zeta^{4}\right) ; \zeta^{1}, \ldots, \zeta^{4}\right] \equiv Z
$$

Also set

$$
s_{i}\left(\zeta^{1}, \ldots, \zeta^{4}\right)=g_{i}\left(\frac{1}{4} \sum_{\nu=1}^{4} \zeta^{\nu} ; \zeta^{1}, \ldots, \zeta^{4}\right) .
$$

Let $\phi$ be a function defined on $\bar{\Omega}_{h}$ and satisfying the boundary condition $\phi_{j}=v_{3}, j=N+1, \ldots, M$. It satisfies the equation $\boldsymbol{L}_{h}\left[\boldsymbol{\phi}_{i}\right]=0, i=1, \ldots, N$ and is a solution of $\boldsymbol{P}_{h}$ if, and only if,

$$
f_{i}\left(\phi_{i} ; \phi_{i 1}, \ldots, \phi_{i 4}\right)=\frac{1}{4} \sum_{\nu=1}^{4} \phi_{i v}, \quad i=1, \ldots, N,
$$

that is, if

$$
\phi_{i}=s_{i}\left(\phi_{i 1}, \ldots, \phi_{i 4}\right), \quad i=1, \ldots, N .
$$

Now let $\phi^{(0)}$ by any function in $\bar{\Omega}_{h}$ satisfying the boundary condition and let the functions $\phi^{(1)}$, $\phi^{(2)}, \ldots$. be defined by either of the two following iteration schemes. (A), set

$$
\begin{aligned}
& \phi_{i}^{(n+1)}=s_{i}\left(\phi_{i 1}^{(n)}, \ldots, \phi_{i 4}^{(n)}\right), \quad i=1, \ldots, N \\
& \phi_{j}^{(n+1)}=\phi_{j}^{(n)}=v_{j}, \quad j=N+1, \ldots, M .
\end{aligned}
$$

(B), set

$$
\phi_{i}^{(n+1)}=s_{i}\left(\tilde{\phi}_{i 1}^{(n)}, \ldots, \tilde{\phi}_{i 4}^{(n)}\right), \quad i=1, \ldots, N,
$$

where

$$
\begin{aligned}
\tilde{\phi}_{i \nu} & = \begin{cases}\phi_{i \nu}^{(n+1)} & \text { if } P_{i \nu}=P_{j} \text { and } j<i, \\
\phi_{i \nu}^{(n)} & \text { otherwise, }\end{cases} \\
\phi_{j}^{(n+1)} & =\phi_{j}^{(n)}=v_{j}, \quad j=N+1, \ldots, M .
\end{aligned}
$$

If the sequence $\left\{\phi^{(n)}\right\}$ converges, then the limit function is a solution of $\boldsymbol{P}_{h}$, for $s_{i}$ is a continuous function of its arguments.

In the case of the Laplace difference equation, (A) corresponds to the difference equation of heat conduction whose solution converges to that of the Laplace equation for $n \rightarrow \infty$ ( $n$ being interpreted as time), and (B) is the well-known Liebmann method.

We assume now that (3) is replaced by the stronger condition

$$
F_{z} \geq \eta>0
$$

and prove that procedure (B) converges. The proof for $(\mathrm{A})$ is practically the same.

By (48)

$$
\phi_{i}^{(n+1)}-\phi_{i}^{(n)}=\sum_{\nu=1}^{4}\left(\frac{\partial s_{i}}{\partial \zeta^{\nu}}\right)^{*}\left(\widetilde{\phi}_{i \nu}^{(n)}-\widetilde{\phi}_{i \nu}^{(n-1)}\right)
$$

where the asterisk indicates that the partial deriva- tives are evaluated at a point $\left(\zeta^{1}, \ldots, \zeta^{4}\right)$,

$$
\zeta^{\nu}=\tau_{i} \widetilde{\phi}_{i \nu}^{(n)}+\left(1-\tau_{i}\right) \widetilde{\phi}_{i \nu}^{(n-1)}, \quad 0 \leq \tau_{i} \leq 1 .
$$

By (41), (44), (45),

$$
\frac{\partial f_{i}}{\partial z} \frac{\partial G}{\partial Z}=1, \quad \frac{\partial f_{i}}{\partial z} \frac{\partial g_{i}}{\partial \zeta^{\nu}}+\frac{\partial f_{i}}{\partial \zeta^{\nu}}=0,
$$

$$
\begin{gathered}
\frac{\partial f_{i}}{\partial \xi^{1}}=-\frac{\partial f_{i}}{\partial \xi^{3}}=\frac{h}{8} F_{p}, \quad \frac{\partial f_{i}}{\partial \xi^{2}}=-\frac{\partial f_{i}}{\partial \xi^{4}}=\frac{h}{8} F_{q}, \\
\frac{\partial s_{i}}{\partial \xi^{\nu}}=\frac{1}{4} \frac{\partial g_{i}}{\partial Z}+\frac{\partial g_{i}}{\partial \xi^{\nu}},
\end{gathered}
$$

so that by $(42)$

$$
\begin{aligned}
& \frac{\partial s_{i}}{\partial \zeta^{1}}=\frac{\frac{1}{4}\left(1-\frac{h}{2} F_{p}\right)}{1+\frac{h^{2}}{4} F_{z}}, \quad \frac{\partial s_{i}}{\partial \zeta^{2}}=\frac{\frac{1}{4}\left(1-\frac{h}{2} F_{q}\right)}{1+\frac{h^{2}}{4} F_{z}}, \\
& \frac{\partial s_{i}}{\partial \zeta^{3}}=\frac{\frac{1}{4}\left(1+\frac{h}{2} F_{p}\right)}{1+\frac{h^{2}}{4} F_{z}}, \quad \frac{\partial s_{i}}{\partial \zeta^{4}}=\frac{\frac{1}{4}\left(1+\frac{h}{2} F_{a}\right)}{1+\frac{h^{2}}{4} F_{z}} .
\end{aligned}
$$

Hence

and setting

$$
\frac{\partial s_{i}}{\partial \zeta^{\nu}} \geq 0
$$

$$
\rho=\frac{1}{1+\frac{h^{2} \eta}{4}}<1,
$$

it follows from (49) that

$$
\sum\left|\frac{\partial s_{i}}{\partial \xi^{\nu}}\right|=\sum \frac{\partial s_{i}}{\partial \zeta^{\nu}} \leq \rho
$$

Setting

$$
m_{n+1}=\max _{\Omega_{h}}\left|\phi^{(n+1)}-\phi^{(n)}\right|,
$$

we conclude from (50) that

$$
m_{n+1} \leq \rho m_{n}
$$

so that

$$
m_{n} \leq \rho^{n-1} m_{1}
$$

and for $p>1$,

$$
\max _{\Omega_{h}}\left|\phi^{(n+p)}-\phi^{(n)}\right| \leq \sum_{\nu=n+1}^{n+p} m_{\nu} \leq \frac{m_{1} \rho^{n}}{1-\rho},
$$

which implies the existence of

$$
\phi=\lim _{n \rightarrow \infty} \phi^{(n)} .
$$

Also, by (46), (48),

$$
\phi_{i}^{n+1}-\phi_{i}=\sum\left(\frac{\partial s_{i}}{\partial \zeta^{\nu}}\right)^{* *}\left(\widetilde{\phi}_{i \nu}^{(n)}-\phi_{i v}\right),
$$


where the double asterisk indicates that the partial derivatives are evaluated at a point $\left(\zeta^{1}, \ldots, \zeta^{4}\right)$,

$$
\zeta^{\nu}=\tau_{i} \widetilde{\phi}_{i \nu}^{(n)}+\left(1-\tau_{i}\right) \phi_{i \nu}, \quad 0 \leq \tau_{i} \leq 1
$$

Hence, setting

$$
\mu_{n+1}=\max _{\Omega_{h}}\left|\phi^{(n+1)}-\phi\right|,
$$

we have that $\mu_{n+1} \leq \rho \mu_{n}$, so that

$$
\mu_{n} \leq \rho^{n} \mu_{0} .
$$

Since $\mu_{0}$ can be estimated by means of (31), we can determine a priori how many steps are needed in order to obtain $\phi$ with a desired degree of accuracy.

Now we drop assumption (49) and set (for $\eta>0$ )

$$
\boldsymbol{L}_{h}^{(\eta)}\left[\phi_{i}\right]=\boldsymbol{L}_{h}\left[\phi_{i}\right]+\eta \phi_{i} .
$$

Let Let $\phi^{(\eta)}$ be the solution of the boundary value problem

$\boldsymbol{L}_{h}^{(\eta)}\left[\phi_{i}\right]=0, \quad i=1, \ldots, N$;

$$
\phi_{j}=v_{j}, \quad j=N+1, \ldots, M .
$$

This solution exists by virtue of the preceding result. Applying (33) with $\boldsymbol{L}_{h}$ replaced by $\boldsymbol{L}_{h}^{(\eta)}$, we conclude that $\left|\phi^{(\eta)}\right| \leq K$, so that $\left|\boldsymbol{L}_{h}\left[\phi_{i}^{(\eta)}\right]\right|=\left|\eta \phi_{i}^{(\eta)}\right| \leq K \eta$. By (31)

$$
\max _{\Omega_{h}}\left|\phi^{\left(\eta^{\prime}\right)}-\phi^{\left(\eta^{\prime \prime}\right)}\right| \leq 2 C K\left(\eta^{\prime}+\eta^{\prime \prime}\right),
$$

so that

$$
\phi=\lim _{\eta \rightarrow \infty} \phi^{(\eta)}
$$

exists. It is clear that $\phi$ is a solution of $\boldsymbol{P}_{h}$. Also, by (31),

$$
\max _{\Omega_{h}}\left|\phi^{(\eta)}-\phi\right| \leq C K \eta .
$$

Since $\phi^{(\eta)}$ can be computed by procedures (A) or (B), we have an effective method of solving $\boldsymbol{P}_{h}$ in all cases.

\section{Extension}

Let $a(x, y), b(x, y), c(x, y)$ be continuous functions defined in $\bar{\Omega}$.

Set

$$
a c-b^{2}>0 \text {. }
$$

$$
\begin{gathered}
\Lambda[\phi]=a \phi_{x x}+2 b \phi_{x y}+c \phi_{y y}, \\
L[\phi]=\Lambda[\phi]-F\left(x, y, \phi, \phi_{x}, \phi_{y}\right) .
\end{gathered}
$$

With this new operator $\boldsymbol{L}$ we can state problem $\boldsymbol{P}$.

Motzkin and Wasow* showed that ( $t$ being a sufficiently large positive integer depending on $\Lambda$ ) $\Lambda$ can be approximated by a difference operator $\Lambda_{h}(h>0)$ defined by

$$
\Lambda_{h}[\phi]=\frac{1}{h^{2}} \sum_{r, s=-t}^{t} \rho^{\tau s}(x, y) \phi(x+r h, y+s h),
$$

*J. Math. Phys. 31 (1953). and satisfying the following conditions:

$\Lambda_{h}[\phi] \equiv \Lambda[\phi]$ if $\phi(x, y)$ is a polynomial of second degree, (59)

$$
\begin{gathered}
\rho^{r s}(x, y)>0 \text { for }(r, s) \neq(0,0), \\
0<\frac{1}{K_{1}} \leq \sum_{(r, s) \neq(0,0)} \rho^{r s}(x, y) \leq K_{1} .
\end{gathered}
$$

Applying (59) to $\phi \equiv 1$ we have that

$$
\rho^{00}(x, y)=-\sum_{(r, s)=(0,0)} \rho^{r s}(x, y)<0 .
$$

If $\phi(x, y)$ has continuous derivatives of the second order whose moduli of continuity do not exceed $\omega^{(2)}(\eta)$, then

$$
\left|\Lambda_{h}[\phi]-\Lambda[\phi]\right| \leq 2 t^{2} K_{1} \omega^{(2)}(\sqrt{2} t h),
$$

and if $\phi(x, y)$ has partial derivatives of the third order bounded in modulus by $m^{(3)}$, then

$$
\left|\Lambda_{h}[\phi]-\Lambda[\phi]\right| \leq \frac{4}{3} t^{3} K_{1} m^{(3)} h .
$$

Under the hypotheses that the fourth order derivatives exist and are bounded by $m^{(4)}$ in modulus this can be improved to

$$
\left|\Lambda_{h}[\phi]-\Lambda[\phi]\right| \leq \frac{2}{3} t^{4} K_{1} m^{(4)} h^{2},
$$

provided $\Lambda_{h}$ satisfies the additional condition

$\Lambda_{h}[\phi] \equiv 0$ if $\phi(x, y)$ is a homogeneous

$$
\text { polynomial of degree } 3 \text {. }
$$

All this follows easily from Taylor's theorem.

Once $\Lambda_{h}$ has been chosen, it is easy to find difference operators

$$
\begin{aligned}
& D_{d, x}[\phi]=\frac{1}{h} \sum_{r, s=-t}^{t} \sigma^{r s}(x, y) \phi(x+r h, y+s h), \\
& D_{h, y}[\phi]=\frac{1}{h} \sum_{r, s=-t}^{t} \tau^{r s}(x, y) \phi(x+r h, y+s h),
\end{aligned}
$$

satisfying the conditions

$$
\begin{gathered}
\sigma^{00}(x, y) \equiv \tau^{00}(x, y) \equiv 0, \\
\left|\sigma^{r s}(x, y)\right|,\left|\tau^{r s}(x, y)\right| \leq K_{2}\left|\rho^{r s}(x, y)\right|,
\end{gathered}
$$

and approximating $\phi_{x}, \phi_{\nu}$ in the sense that

$D_{h, x}[\phi] \equiv \phi_{x}, D_{h, y}[\phi] \equiv \phi_{y}$ for every linear function $\phi(x, y)$.

We have that

$$
\left|D_{h, x}[\phi]-\phi_{x}\right|,\left|D_{h, y}[\phi]-\phi_{y}\right| \leq 2 t K_{1} K_{2} \omega^{(1)}(\sqrt{2} t h)
$$


if $\phi(x, y)$ has continuous partial derivatives with moduli of continuity bounded by $\omega^{(1)}(\eta)$,

$$
\left|D_{h, x}[\phi]-\phi_{x}\right|,\left|D_{h, y}[\phi]-\phi_{y}\right| \leq 2 t^{2} K_{1} K_{2} m^{(2)} h
$$

if the second derivatives exist and are bounded by $m^{(1)}$ in absolute value.

If

$D_{h, x}[\phi] \equiv D_{h, y}[\phi] \equiv 0$ for every homogeneous quadratic polynomial $\phi(x, y)$,

then, under the hypothesis of $\left(63^{\prime}\right)$,

$$
\left|D_{h, x}[\phi]-\phi_{x}\right|,\left|D_{h, y}[\phi]-\phi_{y}\right| \leq \frac{4}{3} t^{3} K_{1} K_{2} m^{(3)} h^{2} .
$$

We approximate $\boldsymbol{L}$ by the difference operator

$$
\boldsymbol{L}_{h}[\boldsymbol{\phi}]=\Lambda_{h}[\phi]-F\left\{x, y, \phi, D_{h, x}[\phi], D_{h, y}[\phi]\right\} .
$$

Let $P_{0}$ be the point $\left(x_{0}, y_{0}\right)$. We order the $T=4 t(t+1)$ points $\left(x_{0}+r h, y_{0}+s h\right)$, where $r, s=0, \pm 1, \ldots, \pm t$, and $(r, s) \neq(0,0)$ lexicographically with respect to $(r, s)$ and denote them by $P_{01}, \ldots, P_{0 T}$. These points shall be called the $h$-neighbors of $P_{0}$. The new definition of neighbors leads to a new definition of the boundary $\Omega_{h}^{\prime}$ of a lattice domain $\Omega_{h}$, and having this new definition, we can state the boundary value problem $\boldsymbol{P}_{h}$ for the operator $\boldsymbol{L}_{h}$ defined by (70).

We make now two remarks concerning the linear difference operator

$$
l_{h}\left[\phi_{i}\right]=\Lambda_{h}\left[\phi_{i}\right]+\alpha_{i} D_{h, x}\left[\phi_{i}\right]+\beta_{i} D_{h, y}\left[\phi_{i}\right]-\gamma_{i} \phi_{i}
$$

subject to conditions (13), (14).

(1) Set

$$
h_{1}=\frac{1}{2 K_{2}} .
$$

Then for $0<h<h_{1}$

$$
l_{h}\left[\phi_{i}\right]=\frac{1}{h^{2}}\left\{\sum_{\nu=1}^{T} \lambda^{i_{\nu}} \phi_{i \nu}-\phi_{i} \sum_{\nu=1}^{T} \lambda^{i_{\nu}}\right\}-\gamma_{i} \phi_{i}
$$

with

$$
\lambda^{i_{\nu}} \geq 0, \quad \sum_{\nu=1}^{T} \lambda^{i_{\nu}}>0 .
$$

In fact, applying (57) to $\phi \equiv 1$, we see that

$$
\Sigma \sigma^{r s}(x, y) \equiv \Sigma \tau^{r s}(x, y) \equiv 0,
$$

so that $l_{h}$ can be written in the form (73) with

$$
\lambda^{i_{\nu}}=\rho^{(r, s)_{\nu}}\left(x_{i \nu}, y_{i v}\right)-h \sigma^{(\tau, s)_{\nu}}\left(x_{i \nu}, y_{i v}\right)-h \tau^{(\tau, s)_{\nu}}\left(x_{i v}, y_{i \nu}\right),
$$

where $P_{i \nu}=\left(x_{i}+r h, y_{i}+s h\right),(r, s)=(r, s)_{\nu} . \quad$ By virtue of (61), (66), condition (72) implies (74).

(2) Let $\widetilde{l}_{h}$ denote the operator (71) with $\gamma_{i} \equiv 0$. There exists a continuous function $\Phi(x, y)$ defined in $\bar{\Omega}$ and positive constants $h_{2}, C$ such that for $0<h<h_{2}$

$$
\frac{\max _{\Omega}^{-} \Phi(x, y)}{\min _{\Omega}^{-} \tilde{l}_{h}[\Phi(x, y)]} \leq C .
$$

In fact, assume (without loss of generality) that $\bar{\Omega}$ is contained in the domain $0 \leq x \leq d$, and set $\Phi \equiv e^{\mu x}, \mu>0$. By $\left(63^{\prime}\right),\left(68^{\prime}\right)$ we have that

$$
\begin{aligned}
\tilde{l}_{h}[\Phi(x, y)] \geq a(x, y) \mu^{2} e^{\mu x}-\frac{4}{3} t^{3} K_{1} \mu^{3} e^{\mu d} h & \\
& -A \mu e^{\mu x}-2 A 2 t^{2} K_{1} K_{2} \mu^{2} e^{\mu d} h .
\end{aligned}
$$

If we first choose a fixed $\mu$ such that

$$
\mu^{2} \min _{\bar{\Omega}} a(x, y)-\mu A>1,
$$

we can determine a $h_{2}$ such that for $h<h_{2} \tilde{l}_{h}[\Phi(x, y)]<\frac{1}{2}$. Then (75) holds with $C=2 e^{\mu d}$.

Using these two remarks and assuming that $h<\min \left(h_{1}, h_{2}\right)$, all considerations of sections 3 to 6 can be repeated with only minor and obvious modifications. Inequality (16) holds with the $C$ determined above, and so does (31). This inequality implies the uniqueness and existence of a solution of $\boldsymbol{P}_{h}$. This solution can be computed effectively by the method of section 5, and its deviation from the solution of $\boldsymbol{P}$ can be estimated as in section 5, using inequalities (63), (68).

We note that the case in which eq (1) is approximated not by (2) but by a more sophisticated difference equation is included in the preceding discussion.

Los Angeles, October 9, 1952. 